\title{
Thermal and frequency stability of dielectric ceramic Ba6-3xNd8+2xTi18O54 (x=0.15, 0.25)
}

\begin{abstract}
A new dielectric material, barium neodymium titanate (BNT) ceramic can provide good thermal and frequency stability on the dielectric properties. The synthesis of BNT ceramics with $\mathrm{x}=0.15$ and 0.25 was carried out using wet solid state method. The ceramics were characterized by X-ray diffraction to identify the phase. The shifting of XRD peaks revealed higher content of neodymium ions inside the compound. Surface morphology of the ceramics was determined using FESEM. Different compositions influenced the grain growth of the ceramics. BNT ceramics with higher neodymium content showed higher porosity, and higher resistance to shrinkage. The dielectric properties at low frequency from $40 \mathrm{~Hz}$ to $1 \mathrm{MHz}$ were measured using Impedance Analyzer. The polarization effect inside the material was discussed and compared. BNT ceramics with $\mathrm{x}=0.15$ has higher dielectric constant. These BNT ceramics showed frequency and thermal stability with respect to the dielectric constant.
\end{abstract}

Keyword: Dielectric properties; Microstructure; Polarization; Thermal stability 